\title{
A LINEAR RECURRENCE SYSTEM
}

\author{
A. BLASIUS
}

(Communicated by William W. Adams)

ABSTRACT. We look at a triangular system of $n$ equations and reinvestigate a related function introduced by Chen and Kuck. Our main contribution is to provide a new proof of a result which forms the basis of their work.

We investigate a function which will be used to evaluate the linear recursion

$$
x_{i}=a_{i, 0}+\sum_{j=1}^{i-1} a_{i, j} x_{j} \quad \text { for } i=1,2,3, \ldots, n
$$

where the $a_{i j}$ 's are arbitrary numbers.

We can express this in matrix notation as $X=C+A X$ where

$$
C=\left[\begin{array}{c}
a_{10} \\
\cdot \\
\cdot \\
\cdot \\
\cdot \\
\cdot \\
a_{n 0}
\end{array}\right], \quad A=\left[\begin{array}{ccccccc}
0 & 0 & 0 & \cdot & \cdot & \cdot & 0 \\
a_{21} & 0 & 0 & \cdot & \cdot & \cdot & 0 \\
a_{31} & a_{32} & 0 & \cdot & \cdot & \cdot & 0 \\
\cdot & \cdot & \cdot & \cdot & & & \cdot \\
\cdot & \cdot & \cdot & & \cdot & & \cdot \\
\cdot & \cdot & \cdot & & & \cdot & \cdot \\
a_{n 1} & a_{n 2} & a_{n 3} & \cdot & \cdot & \cdot & 0
\end{array}\right], \quad X=\left[\begin{array}{c}
x_{1} \\
\cdot \\
\cdot \\
\cdot \\
\cdot \\
\cdot \\
x_{n}
\end{array}\right]
$$

$A$ is referred to as the coefficient matrix, $C$ as the constant vector, and $X$ as the solution vector.

Definition. For $0 \leq j<2^{n}, 1 \leq i \leq 2^{n}$, we define a sequence of functions $f_{0}(i, j), f_{1}(i, j), f_{2}(i, j), \ldots, f_{n}(i, j)$ such that

$$
f_{r+1}(i, j)= \begin{cases}f_{r}(i, j)+\sum_{k=j+2^{r}-b}^{j+2^{r+1}-b-1} f_{r}(i, k) f_{r}(k, j) & \text { if } j \equiv b\left(\bmod 2^{r+1}\right), \\ f_{r}(i, j) \text { otherwise } & \text { where } 0 \leq b<2^{r},\end{cases}
$$

for $0 \leq r<n$, with

$$
f_{0}(i, j)=a_{i, j} \text { and } a_{i, j}=0 \text { for } i \leq j .
$$

Remark. $a_{i, j}=0$ for $i \leq j$ implies $f_{r}(i, j)=0$ for $i \leq j$.

By repeatedly applying this recursive definition we can express any $f_{r+1}(i, j)$ in terms of $f_{r}, f_{r-1}, \ldots$, and finally $f_{0}$, and thus express $f_{r+1}(i, j)$ as a function of $a$ 's only.

Received by the editors November 9, 1992.

1991 Mathematics Subject Classification. Primary 11 B37. 


\section{Examples.}

$$
\begin{aligned}
f_{1}(4,0) & =f_{0}(4,0)+f_{0}(4,1) f_{0}(1,0)=a_{4,0}+a_{4,1} a_{1,0} \\
f_{1}(5,1) & =f_{0}(5,1)=a_{5,1} \\
f_{2}(3,0) & =f_{1}(3,0)+f_{1}(3,2) f_{1}(2,0) \\
& =f_{0}(3,0)+f_{0}(3,1) f_{0}(1,0)+f_{0}(3,2)\left[f_{0}(2,0)+f_{0}(2,1) f_{0}(1,0)\right] \\
& =a_{3,0}+a_{3,1} a_{1,0}+a_{3,2}\left(a_{2,0}+a_{2,1} a_{1,0}\right) .
\end{aligned}
$$

Theorem. Let $j \equiv b\left(\bmod 2^{r}\right), 0 \leq b<2^{r}$; then

$$
f_{r}(i, j)=a_{i, j}+\sum a_{i, j(1)} a_{j(1), j(2)} \cdots a_{j(u), j}
$$

where the sum is over all u-tuples $(j(1), \ldots, j(u))$ satisfying $j<j(u)<\cdots<$ $j(1)<\min \left\{j+2^{r}-b, i\right\}$, where $0<u<\min \left\{2^{r}-b, i-j\right\}$.

Proof. Our proof is by induction on $r$. We observe that

$$
\begin{aligned}
& f_{0}(i, j)=a_{i, j}, \\
& f_{1}(i, j)= \begin{cases}a_{i, j}+a_{i, j+1} a_{j+1, j} & \text { if } j \equiv 0(\bmod 2), \\
a_{i, j} & \text { otherwise. }\end{cases}
\end{aligned}
$$

So the result is verified for $r=0$ and $r=1$.

Assume the result is valid for $r=s$. Now using this, we will deduce the corresponding result for $s+1$ :

$$
f_{s+1}(i, j)=a_{i, j}+\sum a_{i, j(1)} a_{j(1), j(2)} \cdots a_{j(u), j}
$$

where the sum is over all $u$-tuples $(j(1), \ldots, j(u))$ satisfying $j<j(u)<$ $\cdots<j(1)<\min \left\{j+2^{s+1}-b, i\right\}$, where $0<u<\min \left\{2^{s+1}-b, i-j\right\}$ and $j \equiv b\left(\bmod 2^{s+1}\right), 0 \leq b<2^{s+1}$.

We consider three cases.

Case 1. $j \equiv b\left(\bmod 2^{s+1}\right), 2^{s} \leq b<2^{s+1}$. In this case $f_{s+1}(i, j)=f_{s}(i, j)$ by definition, and by the induction hypothesis

$$
\begin{aligned}
f_{s+1}(i, j)=a_{i, j}+ & \sum_{j<j(u)<\cdots<j(1)<\min } a_{i, j(1)} a_{j(1), j(2)} \cdots a_{j(u), j}, \\
& \left.=b^{\prime}, i\right\},
\end{aligned}
$$

where $0<u<\min \left\{2^{s}-b^{\prime}, i-j\right\}$ and $j \equiv b^{\prime}\left(\bmod 2^{s}\right), 0 \leq b^{\prime}<2^{s}$.

But $j \equiv b\left(\bmod 2^{s+1}\right), 2^{s} \leq b<2^{s+1}$, implies $j \equiv b-2^{s}\left(\bmod 2^{s}\right), 0 \leq b<$ $2^{s}$, and $b^{\prime}=b-2^{s}$. Therefore

$$
\begin{aligned}
f_{s+1}(i, j)=a_{i, j}+ & \sum a_{i, j(1)} a_{j(1), j(2)} \cdots a_{j(u), j}, \\
j & <j(u)<\cdots<j(1)<\min \left\{j+2^{s+1}-b, i\right\},
\end{aligned}
$$

where $0<u<\min \left\{2^{s+1}-b, i-j\right\}$ and $j \equiv b\left(\bmod 2^{s+1}\right), 2^{s} \leq b<2^{s+1}$.

Case 2. $j \equiv b\left(\bmod 2^{s+1}\right), 0 \leq b<2^{s}$, and $i \leq j+2^{s}-b$. It follows by definition that $f_{s+1}(i, j)=f_{s}(i, j)$ and by the induction hypothesis

$$
\begin{aligned}
f_{s+1}(i, j)=a_{i, j}+ & \sum a_{i, j(1)} a_{j(1), j(2)} \cdots a_{j(u), j}, \\
& j<j(u)<\cdots<j(1)<\min \left\{j+2^{s}-b^{\prime}, i\right\},
\end{aligned}
$$

where $0<u<\min \left\{2^{s}-b^{\prime}, i-j\right\}$ and $j \equiv b^{\prime}\left(\bmod 2^{s}\right), 0 \leq b^{\prime}<2^{s}$. Since $j \equiv b\left(\bmod 2^{s+1}\right), 0 \leq b<2^{s}$, implies $j \equiv b\left(\bmod 2^{s}\right), 0 \leq b<2^{s}$, we have 
$b^{\prime}=b$. Then $\min \left\{j+2^{s}-b, i\right\}=i=\min \left\{j+2^{s+1}-b, i\right\}$, since $i \leq j+2^{s}-b$. Therefore

$$
\begin{aligned}
f_{s+1}(i, j)=a_{i, j}+\sum a_{i, j(1)} a_{j(1), j(2)} \cdots a_{j(u), j}, \\
j<j(u)<\cdots<j(1)<\min \left\{j+2^{s+1}-b, i\right\},
\end{aligned}
$$

where $0<u<\min \left\{2^{s+1}-b, i-j\right\}$ and $j \equiv b\left(\bmod 2^{s+1}\right), 0 \leq b<2^{s}$, and $i \leq j+2^{s}-b$.

Case 3. $j \equiv b\left(\bmod 2^{s+1}\right), 0 \leq b<2^{s}$, and $i>j+2^{s}-b$. By definition

$$
\begin{aligned}
f_{s+1}(i, j) & =f_{s}(i, j)+\sum_{k=j+2^{s}-b}^{j+2^{s+1}-b-1} f_{s}(i, k) f_{s}(k, j) \\
& =f_{s}(i, j)+\sum_{k=j+2^{s}-b}^{\min \left\{j+2^{s+1}-b, i\right\}-1} f_{s}(i, k) f_{s}(k, j) .
\end{aligned}
$$

From the induction hypothesis

$$
\begin{aligned}
f_{s}(i, k)=a_{i, k}+\sum_{k} a_{i, k(1)} a_{k(1), k(2)} \cdots a_{k(u), k}, \\
k<k(u)<\cdots<k(1)<\min \left\{k+2^{s}-c, i\right\},
\end{aligned}
$$

where $0<u<\min \left\{2^{s}-c, i-k\right\}$ and $k \equiv c\left(\bmod 2^{s}\right), 0 \leq c<2^{s}$. When $j+2^{s}-b \leq k<j+2^{s+1}-b, k=j+2^{s}-b+c$ and $k+2^{s}-c=j+2^{s+1}-b$. Therefore, for all $k$ such that $j+2^{s}-b \leq k<j+2^{s+1}-b$ we have

$$
\begin{aligned}
& f_{s}(i, k)=a_{i, k}+\sum a_{i, k(1)} a_{k(1), k(2)} \cdots a_{k(u), k}, \\
& \quad k<k(u)<\cdots<k(1)<\min \left\{j+2^{s+1}-b, i\right\},
\end{aligned}
$$

where $0<u<\min \left\{j+2^{s+1}-b, i\right\}-k$ and $j \equiv b\left(\bmod 2^{s+1}\right), 0 \leq b<2^{s}$. Also, by the induction hypothesis

$$
\begin{aligned}
\left.f_{s}(k, j)=a_{k, j}+\sum_{j<j(v)<\cdots<j(1)<\min } a_{k, j(1)} a_{j(1), j(2)} \cdots a_{j(v), j}, 2^{s}-b^{\prime}, k\right\},
\end{aligned}
$$

where $0<v<\min \left\{2^{s}-b^{\prime}, k-j\right\}$ and $j \equiv b^{\prime}\left(\bmod 2^{s}\right), 0 \leq b^{\prime}<2^{s}$. But since $j \equiv b\left(\bmod 2^{s+1}\right), 0 \leq b<2^{s}$, it follows that $b^{\prime}=b$, and when $j+2^{s}-b \leq k<j+2^{s+1}-b$ we have $\min \left\{j+2^{s}-b, k\right\}=j+2^{s}-b$. Therefore, for all $k$ such that $j+2^{s}-b \leq k<j+2^{s+1}-b$

$$
\begin{aligned}
& f_{s}(k, j)=a_{k, j}+\sum a_{k, j(1)} a_{j(1), j(2)} \cdots a_{j(v), j}, \\
& j<j(v)<\cdots<j(1)<j+2^{s}-b,
\end{aligned}
$$

where $0<v<2^{s}-b$ and $j \equiv b\left(\bmod 2^{s+1}\right), 0 \leq b<2^{s}$. 
It follows from (i) and (ii) that

$$
\begin{aligned}
& \min \left\{j+2^{s+1}-b, i\right\}-1 \\
& \sum_{k=j+2^{s}-b} f_{s}(i, k) f_{s}(k, j) \\
& =\sum_{k=j+2^{s}-b}^{\min \left\{j+2^{s+1}-b, i\right\}-1}\left(a_{i, k} a_{k, j}+\sum_{j(v)} a_{i, k} a_{k, j(1)} \cdots a_{j(v), j}\right. \\
& +\sum_{k(u)} a_{i, k(1)} \ldots a_{k(u), k} a_{k, j} \\
& \left.+\sum_{k(u), j(v)} a_{i, k(1)} \cdots a_{k(u), k} a_{k, j(1)} \cdots a_{j(v), j}\right)
\end{aligned}
$$

where $k<k(u)<\cdots<k(1)<\min \left\{j+2^{s+1}-b, i\right\}$,

$$
\begin{aligned}
& j<j(v)<\cdots<j(1)<j+2^{s}-b, \\
& 0<u<\min \left\{j+2^{s+1}-b, i\right\}-k, \\
& 0<v<2^{s}-b, \text { and } j \equiv b\left(\bmod 2^{s+1}\right), 0 \leq b<2^{s} .
\end{aligned}
$$

By removing the outer summation symbol and making a change of variables, we obtain

$$
\begin{aligned}
& \sum_{k=j+2^{s}-b}^{\min \left\{j+2^{s+1}-b, i\right\}-1} f_{s}(i, k) f_{s}(k, j) \\
= & \sum_{C(1)} a_{i, j(1)} a_{j(1), j}+\sum_{C(2)} a_{i, j(1)} a_{j(1), j(2)} \cdots a_{j(v+1), j} \\
& +\sum_{C(3)} a_{i, j(1)} \cdots a_{j(u), j(u+1)} a_{j(u+1), j} \\
& +\sum_{C(4)} a_{i, j(1)} \cdots a_{j(u), j(u+1)} a_{j(u+1), j(u+2)} \cdots a_{j(u+v+1), j}
\end{aligned}
$$

where $C(1), C(2), C(3), C(4)$ are the summation conditions:

$C(1): j+2^{s}-b \leq j(1)<\min \left\{j+2^{s+1}-b, i\right\}$ where $j \equiv b\left(\bmod 2^{s+1}\right)$, $0 \leq b<2^{s}$

$C(2): j<j(v+1)<\cdots<j(2)<j+2^{s}-b \leq \min \left\{j+2^{s+1}-b, i\right\}$ where $0<v<2^{s}-b$ and $j \equiv b\left(\bmod 2^{s+1}\right), 0 \leq b<2^{s}$,

$C(3): j+2^{s}-b \leq j(u+1)<j(u)<\cdots<j(1)<\min \left\{j+2^{s+1}-b, i\right\}$ where $0<u<\min \left\{j+2^{s+1}-b, i\right\}-j(u+1)$ and $j \equiv b\left(\bmod 2^{s+1}\right)$, $0 \leq b<2^{s}$,

$C(4): j<j(u+v+1)<\cdots<j(u+2)<j+2^{s}-b \leq j(u+1)<j(u)<\cdots<$ $j(1)<\min \left\{j+2^{s+1}-b, i\right\}$ where $0<u<\min \left\{j+2^{s+1}-b, i\right\}-j(u+1)$, $0<v<2^{s}-b$, and $j \equiv b\left(\bmod 2^{s+1}\right), 0 \leq b<2^{s}$.

Remarks. 1. $u+v+1<\min \left\{2^{s+1}-b, i-j\right\}$.

2. $C(1), C(2), C(3), C(4)$ describe a partition of the condition $j+2^{s}-b \leq j(1)<\min \left\{j+2^{s+1}-b, i\right\}, \quad j<j(w)<\cdots<j(2)<j(1)$, 
where $0<w<\min \left\{2^{s+1}-b, i-j\right\}$ and $j \equiv b\left(\bmod 2^{s+1}\right), 0 \leq b<2^{s}$.

We now conclude

$$
\begin{array}{r}
\sum_{k=j+2^{s}-b}^{\min \left\{j+2^{s+1}-b, i\right\}-1} f_{s}(i, k) f_{s}(k, j)=\sum a_{i, j(1)} \cdots a_{j(w), j}, \\
j+2^{s}-b \leq j(1)<\min \left\{j+2^{s+1}-b, i\right\}, \\
j<j(w)<\cdots<j(2)<j(1),
\end{array}
$$

where $0<w<\min \left\{2^{s+1}-b, i-j\right\}$ and $j \equiv b\left(\bmod 2^{s+1}\right), 0 \leq b<2^{s}$. It follows from the induction hypothesis that

$$
\begin{aligned}
& f_{s}(i, j)=a_{i, j}+\sum a_{i, j(1)} \cdots a_{j(x), j}, \\
& \quad j<j(x)<\cdots<j(1)<\min \left\{j+2^{s}-b^{\prime}, i\right\},
\end{aligned}
$$

where $0<x<\min \left\{2^{s}-b^{\prime}, i-j\right\}$ and $j \equiv b^{\prime}\left(\bmod 2^{s}\right), 0 \leq b^{\prime}<2^{s}$.

We have $j \equiv b\left(\bmod 2^{s+1}\right), 0 \leq b<2^{s}$, therefore $b^{\prime}=b$, and since $i>j+2^{s}-b$ the $\min \left\{j+2^{s}-b, i\right\}=j+2^{s}-b$.

From (iii) and (iv) we conclude that when $j \equiv b\left(\bmod 2^{s+1}\right), 0 \leq b<2^{s}$, and $i>j+2^{s}-b$, then

$$
f_{s+1}(i, j)=a_{i, j}+\sum_{S(1)} a_{i, j(1)} \cdots a_{j(x), j}+\sum_{S(2)} a_{i, j(1)} \cdots a_{j(w), j}
$$

where $S(1)$ and $S(2)$ denote the summation conditions

$$
j<j(x)<\cdots<j(1)<j+2^{s}-b, \quad 0<x<2^{s}-b,
$$

and $j+2^{s}-b \leq j(1)<\min \left\{j+2^{s+1}-b, i\right\}$,

$$
j<j(w)<\cdots<j(2)<j(1), \quad 0<w<\min \left\{2^{s+1}-b, i-j\right\},
$$

respectively. Hence we have

$$
\begin{aligned}
& f_{s+1}(i, j)=a_{i, j}+\sum a_{i, j(1)} a_{j(1), j(2)} \cdots a_{j(y), j}, \\
& \quad j<j(y)<\cdots<j(1)<\min \left\{j+2^{s+1}-b, i\right\},
\end{aligned}
$$

where $0<y<\min \left\{2^{s+1}-b, i-j\right\}$ and $j \equiv b\left(\bmod 2^{s+1}\right), 0 \leq b<2^{s}$, and $i>j+2^{s}-b$.

Together, Cases 1, 2, and 3 show that the theorem holds for $r=s+1$, and thus for all nonnegative integers less than or equal to $n$.

Corollary. $f_{n}(i, 0)=a_{i, 0}+\sum_{j=1}^{i-1} a_{i, j} x_{j}=x_{i}$ for $1 \leq i \leq 2^{n}$.

Proof. Our proof is by induction on $i$. The corollary is true for $i=1$ and $i=2$, since $f_{n}(1,0)=a_{1,0}=x_{1}$ for $n \geq 0$ and $f_{n}(2,0)=a_{2,0}+a_{2,1} a_{1,0}=x_{2}$ for $n \geq 1$. As an induction hypothesis, assume it holds for all $i \leq k<2^{n}$. Thus, by hypothesis we have

$$
f_{n}(i, 0)=a_{i, 0}+\sum_{j=1}^{i-1} a_{i, j} x_{j}=x_{i} \quad \text { for } 1 \leq i \leq k .
$$

Now we prove the lemma for $i=k+1$. We know by definition that

$$
x_{k+1}=a_{k+1,0}+\sum_{j=1}^{k} a_{k+1, j} x_{j},
$$


and by the induction hypothesis this can be written as

$$
x_{k+1}=a_{k+1,0}+\sum_{j=1}^{k} a_{k+1, j} f_{n}(j, 0) \text {. }
$$

It follows from our theorem that

$$
\begin{aligned}
& x_{k+1}=a_{k+1,0}+\sum_{j=1}^{k} a_{k+1, j}\left(a_{j, 0}+\sum a_{j, j(1)} a_{j(1), j(2)} \cdots a_{j(u), 0}\right), \\
& \quad 0<j(u)<\cdots<j(1)<\min \left\{2^{n}, j\right\}, \quad 0<u<\min \left\{2^{n}, j\right\},
\end{aligned}
$$

and we obtain

$$
\begin{aligned}
x_{k+1}=a_{k+1,0}+ & \sum_{\substack{1 \leq j(1) \leq k \\
0<j(u+1)<\cdots<j(2)<j(1) \leq k, \quad}} a_{k+1, j(1)} a_{j(1), 0}+\sum a_{k+1, j(1)} \cdots a_{j(u+1), 0}, \\
& 0<u<j(1) .
\end{aligned}
$$

Therefore,

$$
\begin{aligned}
x_{k+1}=a_{k+1,0}+ & \sum_{0<j(v)<\cdots<j(1) \leq k, \quad} a_{(k+1), j(1)} \cdots a_{j(v), 0}, \\
& 0<v \leq k,
\end{aligned}
$$

which can be written as

$$
\begin{gathered}
x_{k+1}=a_{k+1,0}+\sum a_{k+1, j(1)} \cdots a_{j(v), 0}, \\
0<j(v)<\cdots<j(1)<\min \left\{2^{n}, k+1\right\}, \quad 0<v<\min \left\{2^{n}, k+1\right\},
\end{gathered}
$$

and it follows by our theorem that

$$
x_{k+1}=f_{n}(k+1,0) \text {. }
$$

Based on the result that $f_{n}(i, 0)=x_{i}$ for $1 \leq i \leq 2^{n}$ Chen and Kuck [CK] give a parallel algorithm for evaluating $x_{i}, 1 \leq i \leq 2^{n}$. Time and processor bounds for solving the linear recurrence system are then obtained. Sameh and Brent [SB] later presented an alternate derivation of this algorithm.

\section{ACKNOWLEDGMENT}

This paper is an abstract of the author's doctoral thesis, completed under the guidance of David Lubell at Adelphi University.

\section{REFERENCES}

[B] A. Blasius, Parallel processing of linear recurrence systems, Rep. No. 8712611, Ph.D. Thesis, Department of Mathematics and Computer Science, Adelphi University, New York, 1987.

[CK] S. C. Chen and D. J. Kuck, Time and parallel processor bounds for linear recurrence systems, IEEE Trans. Comput. C-24 (1975), 701-717.

[K] D. J. Kuck, Parallel processing of ordinary programs, Advances in Computers (M. Rubinoff and M. C. Yovits, eds.), vol. 15, Academic Press, New York, 1976, pp. 119-179.

[SB] A. H. Sameh and R. P. Brent, Solving triangular systems on a parallel computer, SIAM J. Numer. Anal. 14 (1977), 1101-1113.

Department of Mathematics, SUNY, College at Old Westbury, Old Westbury, New YORK 11568

E-mail address: blasiusa@snyoldva 